\title{
Eine schwierige Diagnose für die ganze Familie
}

\begin{abstract}
Die Diagnose Typ-1-Diabetes ist nicht nur für das betroffene Kind, sondern für die gesamte Familie eine Herausforderung, die eine Lebensumstellung erfordert. Mit der 2. Auflage des Taschenbuchs "Diabetes bei Kindern“ steht jetzt wieder ein aktueller Ratgeber zur Unterstützung der Eltern zur Verfügung, damit zusammen mit den behandelnden Ärzten eine optimale Versorgung erreicht wird.
\end{abstract}

Das im Jahr 2002 erstmals erschienene Buch „Diabetes bei Kindern“ lieferte eine realistische Darstellung der Situation pädiatrischer Patienten mit Typ-1-Diabetes -mellitus und konnte Eltern damit wirkungsvoll unterstützen, wie Dipl.-Psych. Béla Bartus vom Olgahospital in Stuttgart gesagt hat. Leider war dieser Ratgeber bereits seit langem vergriffen. Erst jetzt ist mit Unterstützung des Unternehmens Lilly Diabetes eine komplett überarbeitete Neuauflage erhältlich (TRIAS Verlag, Stuttgart 2012).

Das Buch wird laut Bartus nicht nur von Eltern, sondern auch von Kinder-Diabetologen geschätzt, da es sehr anwendungsbezogen geschrieben ist. Dargestellt werden sowohl medizinische als auch psychologische Aspekte der Erkrankung und die unterschiedlichen Herausforderungen bei pädiatrischen und heranwachsenden $\mathrm{Pa}$ tienten.

\section{Große Fortschritte bei der Therapie in nur einer Dekade}

Die Überarbeitung des Ratgebers war erforderlich, da sich die Therapie bei Typ1-Diabetes seit der Jahrtausendwende dramatisch verändert hat. So wurde die Insulintherapie deutlich intensiviert, entweder in Form der intensivierten konventionellen Insulintherapie (ICT) oder als Pumpentherapie. Vor allem Kleinkinder erhalten heute bereits sofort bei der Dia- gnosestellung oder kurz danach eine Insulinpumpe, so Dr. Martin Holder, ebenfalls vom Olgahospital in Stuttgart.

\section{Turboinsuline machen}

\section{Alltagsgestaltung flexibler}

Als großen Fortschritt in der Insulintherapie wertete Holder die Einführung kurz wirksamer Analoginsuline wie dem Insulin lispro (Humalog ${ }^{\circledR}$ ), die 2010 bereits von etwa $60 \%$ der pädiatrischen Patienten mindestens einmal täglich injiziert wurden. Denn diese „Turboinsuline", so Holder, haben bei Kindern, deren Mahlzeiten und Nahrungsaufnahme schlecht planbar sind, besondere Vorteile: So wird wegen der im Vergleich zu Normalinsulin rascheren Anflutung und der kürzeren Wirkdauer der modernen Insuline kein Spritz-Ess-Abstand benötigt. Möglich ist auch die Insulingabe nach einer Mahlzeit, sodass die Dosis genau auf die zugeführte Nahrungsmenge abgestimmt werden kann. Damit werden Kinder wie Eltern wieder flexibler und sicherer in der Alltagsgestaltung. Bericht: Dr. Katharina Arnheim, Freiburg

Quelle: Pressegespräch „Typ-1-Diabetes: Die Kleinsten benötigen die größte Flexibilität" am 17.5.2012 bei der DDG-Jahrestagung in Stuttgart; Veranstalter: Lilly
Menarini-Preis 2012 an ging an Dr. Knut Mai

Der mit 15000 Euro dotierte MenariniPreis für herausragende wissenschaftliche Forschungsprojekte auf dem Gebiet des Diabetes mellitus, gestiftet von Berlin-Chemie, ging in diesem Jahr an Dr. med. Knut Mai von der Charité Berlin. Der Oberarzt an der Medizinischen Klinik für Endokrinologie, Diabetes und Ernährungsmedizin erforscht die $\mathrm{Zu}$ sammenhänge zwischen dem subkutanen Fettgewebe und Diabetes.

Mit dem Preisgeld möchte Mai die regionale Steroidaktivität in unterschiedlichen subkutanen Fettgewebsdepots genauer analysieren und prüfen, inwiefern Assoziationen mit Parametern der Insulinsensitivität bestehen. Bereits in früheren Untersuchungen konnte Mai mit seinen Kollegen zeigen, dass der lokale Steroidstoffwechsel eng mit Veränderungen der Insulinsensitivität assoziiert ist [1]

1. Mai K et al. Clin Endocrinol (Oxf) 2007; (67): 419ff.

Quelle: Berlin-Chemie, Preisverleihung beim DDG-Kongress am 17.5.2012, Stuttgart

\section{Kleiner Leitfaden zu korrekten Insulininjektion}

Eine achtseitige, praktische Anleitung von Becton, Dickinson and Company (BD), den „Kleiner Leitfaden zur sicheren und sanften Insulininjektion “ dient als Informationsquelle für eine zuverlässige und problemlose Selbstinjektion von Insulin in das Unterhautgewebe. Mithilfe der anschaulichen Broschüre können die häufigsten Fehler und Probleme dabei vermieden werden. Kernthema ist die korrekte Durchführung einer Insulininjektion. Hier werden alle Schritte vorgestellt und erläutert. Hinweise zur Hygiene bei der Injektion, der Einmalverwendung von PenNadeln, dem richtigen Umgang mit Insulin sowie dem sicheren Entsorgen gebrauchter Kanülen vervollständigen den Inhalt.

Der Leitfaden ist auch in Russisch und Türkisch zum Herunterladen bereit unter: www.bddiabetes.de unter „Informationen und Tipps/Downloads/ Injektionstechnik“.

Quelle: BD 